\title{
Argus: A 16-pixel Millimeter-Wave Spectrometer for the Green Bank Telescope
}

\author{
Matthew Sieth ${ }^{a, b}$, Kiruthika Devaraj ${ }^{a, b}$, Patricia Voll ${ }^{a, b}$, Sarah Church $^{a, b}$, Rohit Gawande ${ }^{c}$, Kieran \\ Cleary $^{c}$, Anthony C.S. Readhead ${ }^{c}$, Pekka Kangaslahti ${ }^{d}$, Lorene Samoska ${ }^{d}$, Todd Gaier ${ }^{d}$, Paul F. \\ Goldsmith $^{d}$, Andrew I. Harris ${ }^{e}$, Joshua O. Gundersen ${ }^{f}$, David Frayer ${ }^{g}$, Steve White ${ }^{g}$, Dennis Egan $^{g}$, \\ Rodrigo Reeves ${ }^{h}$ \\ ${ }^{a}$ Stanford University, Department of Physics, 382 Via Pueblo Mall, Stanford, CA 94305, U.S.A.; \\ ${ }^{b}$ Kavli Institute for Particle Astrophysics \& Cosmology, \\ MS 29, Stanford, CA 94309, U.S.A.; \\ ${ }^{c}$ California Institute of Technology, Department of Astronomy, \\ 1200 East California Boulevard, Pasadena, CA 91125, U.S.A.; \\ ${ }^{d}$ Jet Propulsion Laboratory, California Institute of Technology, \\ 4800 Oak Grove Drive, Pasadena, CA 91109, U.S.A.; \\ ${ }^{e}$ University of Maryland, Department of Astronomy, College Park, MD 20742, U.S.A.;

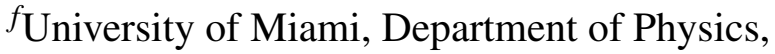 \\ 1320 Campo Sano Drive, Coral Gables, FL, 33146, U.S.A.; \\ ${ }^{g}$ National Radio Astronomy Observatory, P.O. Box 2, Green Bank, WV 24944, U.S.A; \\ ${ }^{h}$ CePIA, Departamento de Astronomìa, Universidad de Concepción, \\ Casilla 160-C, Concepción, Chile.
}

\begin{abstract}
We report on the development of Argus, a 16-pixel spectrometer, which will enable fast astronomical imaging over the 85-116 GHz band. Each pixel includes a compact heterodyne receiver module, which integrates two InP MMIC low-noise amplifiers, a coupled-line bandpass filter and a sub-harmonic Schottky diode mixer. The receiver signals are routed to and from the multi-chip MMIC modules with multilayer high frequency printed circuit boards, which includes LO splitters and IF amplifiers. Microstrip lines on flexible circuitry are used to transport signals between temperature stages. The spectrometer frontend is designed to be scalable, so that the array design can be reconfigured for future instruments with hundreds of pixels. Argus is scheduled to be commissioned at the Robert C. Byrd Green Bank Telescope in late 2014. Preliminary data for the first Argus pixels are presented.
\end{abstract}

Keywords: millimeter-wave, spectrometer, cryogenic focal plane array, W-band, InP MMIC low-noise amplifiers, multichip modules, star formation

\section{INTRODUCTION}

Molecular spectroscopy at millimeter and submillimeter wavelengths allows for detailed studies of the structure and dynamics of molecules in star forming regions. Historically, spectroscopic imaging has generally been implemented either over small areas of the sky at high angular resolution or over large areas at moderate to low angular resolution. Imaging low surface brightness emission over large areas of sky and at high angular resolution is demanding and requires an instrument

Further author information: (Send correspondence to M.S.)

M.S.: E-mail: mmsieth@stanford.edu

Millimeter, Submillimeter, and Far-Infrared Detectors and Instrumentation for Astronomy VII, edited by Wayne S. Holland, Jonas Zmuidzinas, Proc. of SPIE Vol. 9153, 91530P - (c) 2014

SPIE · CCC code: $0277-786 X / 14 / \$ 18 \cdot$ doi: $10.1117 / 12.2055655$ 
with sufficient sensitivity. The Rayleigh-Jeans sensitivity, $\Delta T_{\min }$, for a spectral line observation of a region much larger than the telescope beam solid angle is:

$$
\Delta T_{\min } \propto \frac{T_{\text {sys }}}{\sqrt{\Delta \nu \tau}}
$$

where $\Delta T_{\min }$ is the equivalent temperature of the minimum detectable signal in a spectral channel, $T_{\text {sys }}$ is the system noise temperature of a receiver, which is the sum of the receiver noise temperature and atmospheric contributions to the system noise, $\Delta \nu$ is the spectral resolution, and $\tau$ is the integration time for the observation. The mapping speed for imaging a large region scales approximately with $n_{\text {pix }} /\left(\Delta T_{\min }\right)^{2}$ where $n_{\text {pix }}$ is the number of pixels.

The path to improved mapping speed is therefore to build focal plane arrays with a large number of pixels and low system temperatures. Recent advances in Monolithic Millimeter-wave Integrated Circuit (MMIC) technology ${ }^{1-4}$ have facilitated the construction of large-format focal plane arrays with low system temperatures. Pioneering instruments using MMIC technology have imaged carbon monoxide (CO) and its isotopologues at high angular resolution and over large areas of sky in order to probe the physics and chemistry of molecular clouds. ${ }^{5-7}$ The Argus instrument, which is described herein, builds upon the receiver developments in experiments such as the $\mathrm{Q} / \mathrm{U}$ Imaging ExperimenT (QUIET) ${ }^{8}$ to achieve state-of-the-art mapping speeds.

Argus is a 16-pixel spectrometer that will deploy to the $100 \mathrm{~m}$ Robert C. Byrd Green Bank Telescope (GBT) in late 2014. The instrument capabilities are summarized in Table 1. Argus is designed for the $85-116 \mathrm{GHz}$ band, which includes the astronomically important ${ }^{12} \mathrm{CO}(1-0)$ and $\mathrm{HCN}(1-0)$ lines. It will achieve record mapping speeds for a millimeter-wave imager with receiver temperatures expected to be less than $53 \mathrm{~K}$ per pixel. The GBT will provide an angular resolution of about 7" at $100 \mathrm{GHz}$. This is sufficient resolution to resolve star-forming cores in the Milky Way, which are the smallest units of star formation, and molecular clouds in nearby galaxies. This resolution also overlaps with the angular scales from interferometric arrays such as CARMA, PdBI, and ALMA, which have superior angular resolutions but cannot resolve extended emission. It will therefore be possible to combine Argus data with interferometric datasets to attain much greater spacial dynamic range.

\begin{tabular}{|c|c|}
\hline \multicolumn{2}{|c|}{ Argus Specifications } \\
\hline Number of Pixels & 16 \\
\hline Frequency Range & $85-116 \mathrm{GHz}$ \\
\hline Receiver Temperature & $<53 \mathrm{~K}$ per pixel \\
\hline Angular Resolution & 7' at $100 \mathrm{GHz}$ \\
\hline
\end{tabular}

Table 1. Specifications for the Argus array

This paper is organized as follows: $\S 2$ describes the primary science goals for Argus, $\S 3$ describes the instrument requirements and design, and $\S 4$ describes preliminary lab testing of parts of the array.

\section{ARGUS SCIENCE}

The authors' primary science goal with Argus is to study star formation processes in the Milky Way and nearby galaxies through millimeter-wave molecular spectroscopy. The most abundant molecule in molecular clouds is $\mathrm{H}_{2}$. However, its rotational transitions require high temperatures to excite due to its low mass and so it is mostly invisible in cold molecular clouds. Therefore other molecules must be observed as tracers of molecular gas. Table 2 gives a list of some of the key molecular spectral lines that will be visible in the Argus passband. Carbon monoxide (CO) is the next most abundant molecule after $\mathrm{H}_{2}$ and its lines are the brightest; $\mathrm{CO}$ and its isotopologues are used as tracers of the total molecular gas content and reveal the overall structure and dynamics of molecular clouds. Emission from molecules with higher dipole moments (e.g. $\mathrm{HCN}, \mathrm{HCO}^{+}, \mathrm{HNC}, \mathrm{N}_{2} \mathrm{H}^{+}$, and CS) traces dense molecular gas and can probe the protostellar cores within molecular clouds.

Argus observations will uncover the relationships of the various molecular lines to star formation. It has been shown that $\mathrm{HCN}$, which traces dense gas cores, correlates more closely with star formation than $\mathrm{CO} .{ }^{9}$ It is therefore possible to distinguish starburst modes of star formation by identifying areas with high $\mathrm{HCN} / \mathrm{CO}$ ratios. Argus observations will allow for the specific star formation rate to be parameterized in terms of $\mathrm{CO}, \mathrm{HCN}$, and other tracers for different galaxy types. Since Argus will map large areas of sky at high angular resolution, it will also be possible to understand the links 


\begin{tabular}{|l|r|r||l|c|r|}
\hline Species & Transition & Freq. [GHz] & Species & Transition & Freq. [GHz] \\
\hline $\mathrm{H}^{13} \mathrm{CN}$ & $1-0$ & 86.340 & ${ }^{13} \mathrm{CS}$ & $2-1$ & 92.494 \\
$\mathrm{H}^{13} \mathrm{CO}^{+}$ & $1-0$ & 86.754 & $\mathbf{N}_{2} \mathbf{H}^{+}$ & $1-0$ & 93.173 \\
$\mathrm{SiO}^{1}$ & $2-1$ & 86.847 & $\mathrm{C}^{34} \mathrm{~S}$ & $2-1$ & 96.413 \\
$\mathrm{HN}^{13} \mathrm{C}$ & $1-0$ & 87.091 & $\mathbf{C S}$ & $2-1$ & 97.981 \\
$\mathbf{C}_{2} \mathbf{H}$ & $1-0$ & 87.317 & $\mathrm{C}^{18} 0$ & $1-0$ & 109.782 \\
$\mathbf{H C N}$ & $1-0$ & 88.632 & ${ }^{13} \mathrm{CO}$ & $1-0$ & 110.201 \\
$\mathrm{H}^{15} \mathrm{NC}$ & $1-0$ & 88.866 & $\mathrm{C}^{17} 0$ & $1-0$ & 112.359 \\
$\mathbf{H C O}^{+}$ & $1-0$ & 89.189 & $\mathbf{C N}$ & $1-0$ & 113.491 \\
$\mathbf{H N C}$ & $1-0$ & 90.664 & $\mathbf{C O}$ & $1-0$ & 115.271 \\
\hline
\end{tabular}

Table 2. List of some key transitions from $85-116 \mathrm{GHz}$. Names of the principal isotopic species are in bold.

between large scale processes in the interstellar medium and dense protostellar cores. Such detailed information about star formation in the Milky Way is critical for understanding galaxy-scale star formation through the universe and throughout cosmic time.

Argus will also be well suited for studying comets and astrobiology. Comets are difficult to measure well with an interferometer because of they rotate too quickly. A large-format focal plane array like Argus will enable the molecular content of comets to be imaged rapidly, which will uncover information about the history of the solar system. Observing the interstellar medium with Argus may reveal simple sugars like glycolaldehyde and ethylene glycol, which are important for understanding the conditions from which life forms.

\section{ARGUS INSTRUMENT DESCRIPTION}

\subsection{Instrument Design}

Argus is designed with 16 pixels, a number that was chosen to match the number of channels available in the GBT backend. The pixels are arranged in a $4 \times 4$ array configuration with a spacing of approximately $4.9 F \lambda$ at $100 \mathrm{GHz}$. The array is comprised of four sub-units, or cards, that are installed into a comb-like card cage as shown in Figure 1(a). Two 4-pixel card designs are presented: a prototype card designed to have wide instantaneous bandwidth and a production card that has a tunable $1.5 \mathrm{GHz}$ bandwidth to match the backend capabilities of the GBT. Both designs cover the astronomically rich $85-116 \mathrm{GHz}$ band. However, the smaller instantaneous bandwidth in the production design allowed for a number of simplifications described further in $\$ 3.4 .1$. Concept drawings of the prototype and production designs are shown in Figures 1(b) and 1(c), respectively.

In both the prototype and the production design, a card includes four complete heterodyne receivers. Figure 2 shows a circuit schematic for an Argus pixel. A feedhorn (\$3.2) couples incoming radiation to a miniaturized receiver module (§3.3) that amplifies and downconverts the RF signal. The receiver modules leverage MMIC technology, which permits the integration of several receiver components into a single compact block. The MMIC receiver modules plug into a cryogenic multilayer printed circuit board (§3.4.1) that routes the IF, LO, and DC signals to and from the modules. Flexible circuitry (§3.4.2) is used to transport high densities of IF and DC signals between temperature stages.

Argus is a demonstration of receiver technology that is both highly scalable and capable of observing over extremely wide bandwidths. The 4-pixel cards could in principle be used as the building blocks for a future array with hundreds of pixels. Additionally, the instrument sub-components were designed to be mass-producible. Therefore, while the 16-pixel Argus array will be a powerful tool in itself for molecular spectroscopy, it also serves as a proof-of-concept for even more powerful future instruments.

\subsection{Feeds}

Smooth-walled feedhorns have been developed for Argus that can be economically mass-produced. Feedhorns were chosen instead of a planar antenna design, which would be innately mass-producible, in order to achieve the best possible bandwidth and pixel-to-pixel isolation. The feedhorn design is a modified version of that presented in [10]. The feedhorn profile is a spline with monotonically increasing diameter so that it can be easily machined with either a CNC milling machine or drilled with a mandrel. The beam pattern of these feedhorns is comparable to that of corrugated horns in terms of symmetry and sidelobe level, despite being lighter and easier to manufacture. The sidelobe level is more than $20 \mathrm{~dB}$ below the on-axis response over the entire Argus band. 


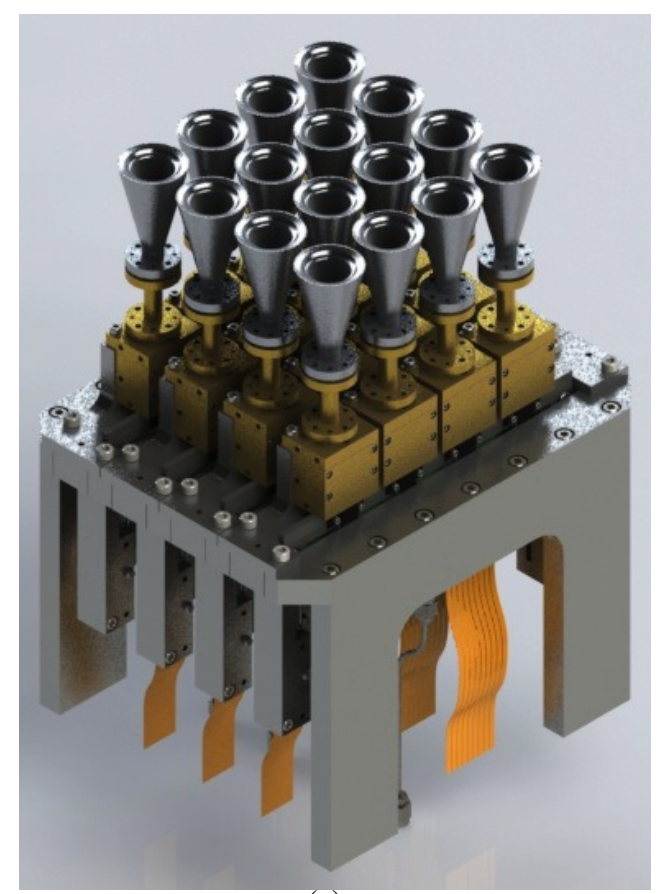

(a)

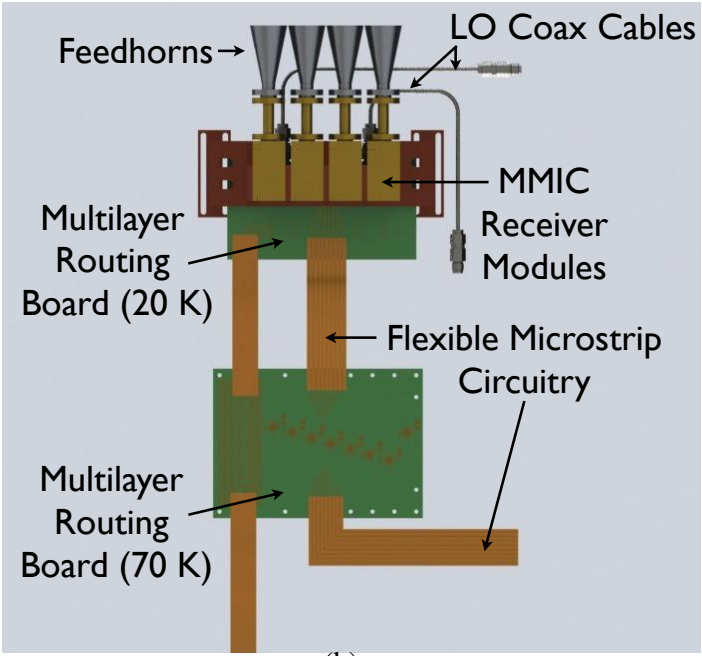

(b)

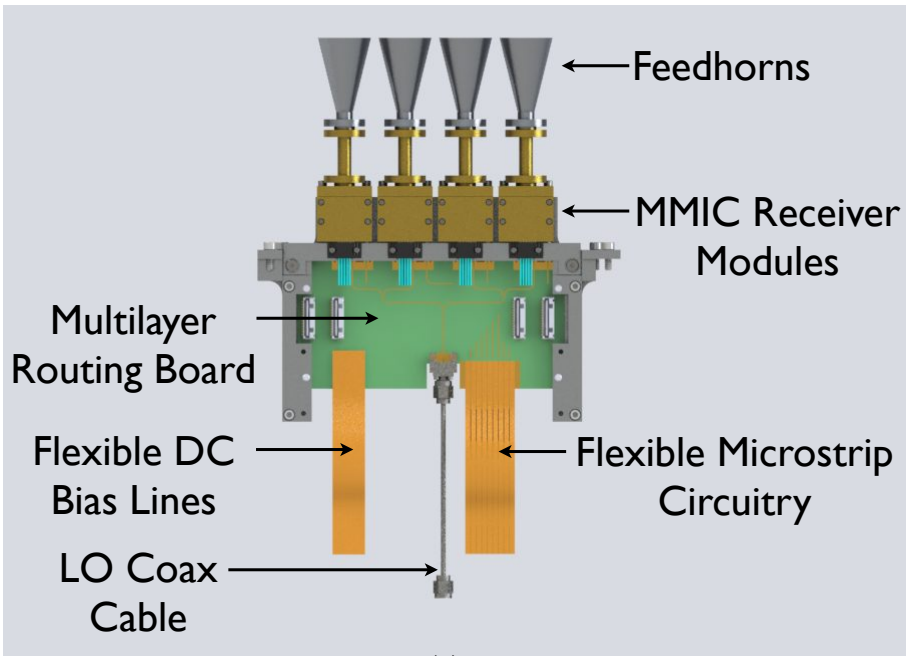

(c)

Figure 1. (a) A 3-D CAD drawing of the 4x4 Argus focal plane array. The array is built from 4-pixel subunits, or cards, which form the rows of the array. A card comprises the feedhorns, MMIC receiver modules, routing boards, flexible circuitry and cables. An annotated 3-D CAD drawing of (b) the prototype 4-pixel card and (c) the production version are shown. The production card has a $1.5 \mathrm{GHz}$ instantaneous bandwidth (to match the GBT backend), while the prototype card was designed for wider instantaneous bandwidth. The IF amplifier used in the prototype card dissipates too much power to be placed on the $20 \mathrm{~K}$ stage and was therefore was incorporated onto a second multilayer routing board on the $70 \mathrm{~K}$ stage. 


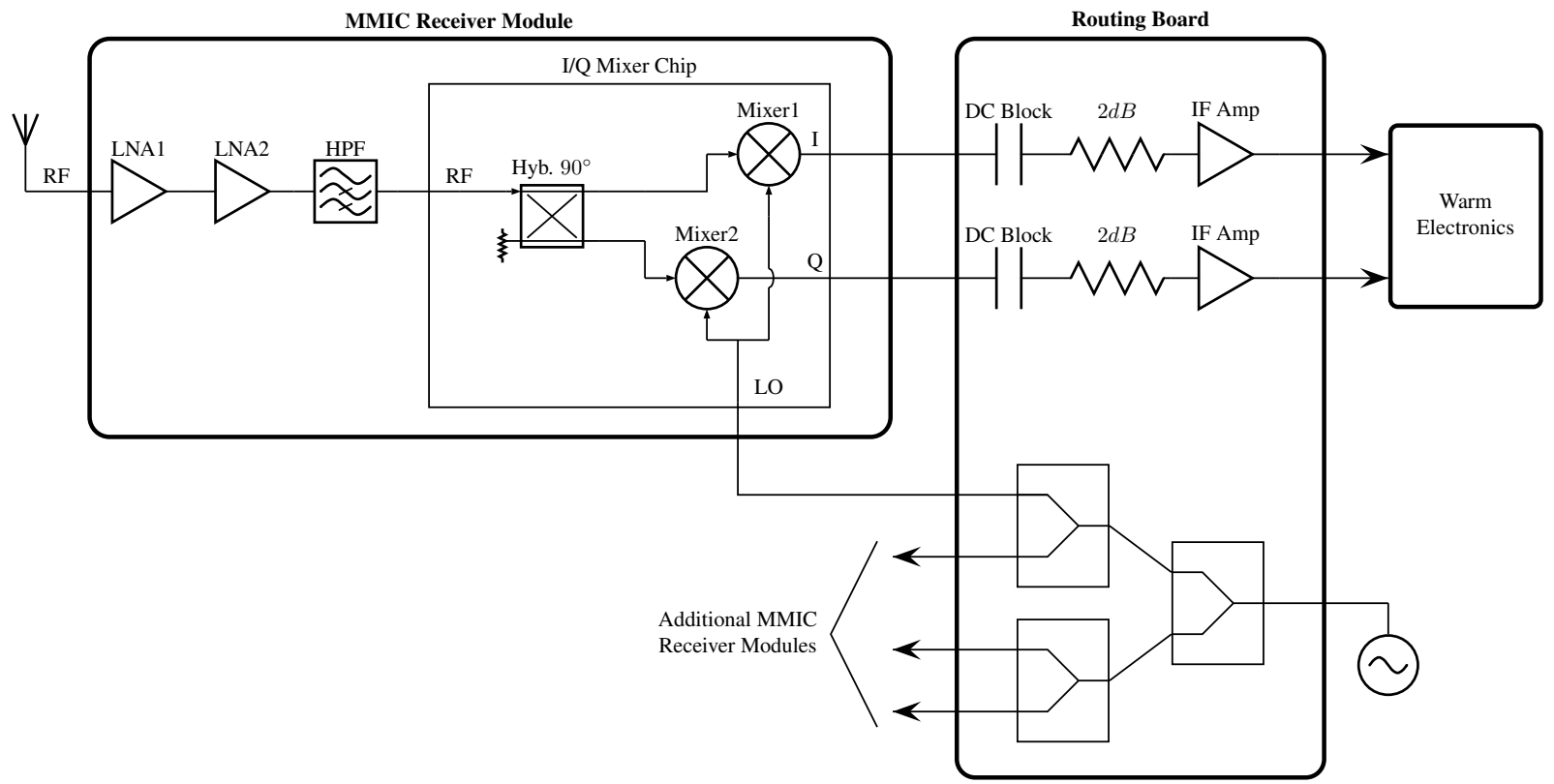

Figure 2. Schematic of an Argus pixel. The MMIC receiver module amplifies, filters, and downconverts the incoming signal. The receiver module attaches to a multilayer routing board with snap-on connectors. The routing board transfers the IF, LO, and DC signals to and from the receiver module and also provides IF amplification and LO splitting. The signals are routed between temperature stages with flexible circuitry.

\subsection{MMIC Receiver Modules}

Each pixel includes an ultra-low noise miniaturized heterodyne receiver whose small footprint is suitable for tight packing in a large-format focal plane array. The miniaturized receivers integrate multiple MMIC components into a single compact module including a waveguide-to-coplanar waveguide probe transition, ${ }^{11}$ two low noise amplifiers (\$3.3.2), a filter, an equalizer, and an I/Q mixer (§3.3.3). These parts are packaged in a brass block that is gold-plated to reduce loss. A prototype version of the MMIC receiver module exhibited a noise temperature of $27-45 \mathrm{~K}$ over the $85-116 \mathrm{GHz}$ frequency band with a gain of more than $20 \mathrm{~dB} .^{12}$ Photographs of the MMIC receiver module are shown in Figure 3 . The production version of the MMIC module has a similar design but is more compact.

\subsubsection{Packaging}

The MMIC receiver modules utilize a split-block package, which is hermetic and provides high isolation between components. The components are installed in a base block while the two other pieces form lids for the RF and DC circuitry, respectively. An RF lid provides a hermetic seal in conjunction with pressure ridges along the RF path in the base block. When joined, the base block and RF lid form a WR-10 waveguide input at their interface. The RF components are mounted in 0.015 " deep cavities in the base block using silver epoxy*. The components are then interconnected with gold ribbon wire bond. While the Argus MMIC receiver modules are hand-assembled, the split-block concept is conducive to automated assembly via pick-and-place machines and automatic wire bonding machines.

The MMIC receiver modules are designed to be individually removable to allow for broken or poorly performing modules can be swapped without having to remove an entire card. Since the pixels are closely packed, the installation and removal of modules must be done blindly. The blind mate was enabled by two design decisions: a length-wise keying mechanism for module placement and the use of push-on connectors. The keying mechanism was machined with wire EDM and is shown in Figure 3. The IF and LO are routed through a high-frequency push-on connector ${ }^{\dagger}$. The connectors are rated up to $60 \mathrm{GHz}$, which is above the maximum LO frequency for Argus.

${ }^{*}$ EPO-TEK H20E

${ }^{\dagger}$ A custom Corning Gilbert GPPO assembly 


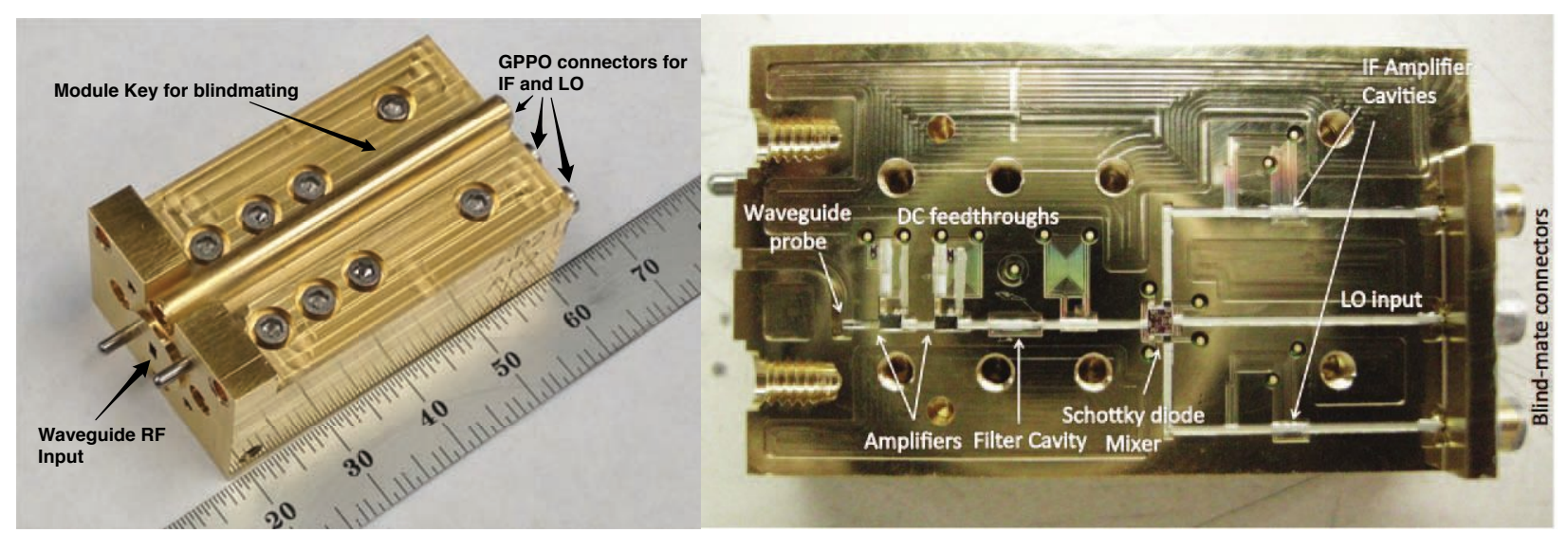

Figure 3. (a) A photograph of a prototype MMIC receiver module. (b) The interior of the MMIC receiver module with components labeled. The circuits are epoxied into RF cavities and then interconnected with gold ribbon wire bonds.

On the bottom side of the base block, a printed circuit board routes the bias voltages into the receiver and includes protection circuitry and resistive dividers. Hermetic DC feedthroughs ${ }^{\ddagger}$ pass the bias voltages from the DC side of the base block to the RF side. A compact 9-pin micro-miniature strip connector ${ }^{\S}$ is used to connect the bias voltage to the MMIC module. These connectors are also amenable to push-on assembly.

\subsubsection{Low-noise amplifiers}

The low-noise amplifiers are three-stage devices that were manufactured by Northrop Grumman Corporation using a $35 \mathrm{~nm}$ Indium Phosphide (InP) high electron mobility transistor (HEMT) process. ${ }^{4}$ These devices are notable for their exceptionally wide bandwidth. The amplifiers were measured to have a noise temperature of less than $40 \mathrm{~K}$ across the entire Argus band and a record low spot noise temperature of $23 \mathrm{~K}$ at $108 \mathrm{GHz}$. Furthermore, the gain of these amplifiers over the Argus band is $22-27 \mathrm{~dB}$. The devices are screened at the chip level with a cryogenic probe station ${ }^{13}$ before being installed into MMIC receiver modules.

\subsubsection{Mixer}

The RF signal is down-converted to an intermediate frequency (IF) of 1.0-2.5 GHz by a subharmonically pumped I/Q mixer. ${ }^{14}$ The mixer was implemented with an antiparallel GaAs Schottky diode pair. In the I/Q scheme, which is illustrated in Figure 2, both the upper and lower sidebands from the downconversion are carried on the in-phase (I) and quadraturephase (Q) IF outputs; the sidebands can be separated off-chip by combining the I and Q outputs with a quadrature hybrid. Subharmonic mixers are preferred to fundamental mixers for this application because the required LO is lower in frequency and therefore easier to route; the LO frequency in Argus is tunable over the 40-58 GHz band. The conversion loss of the mixers is $12-18 \mathrm{~dB}$ across the Argus band at $10 \mathrm{dBm}$ LO power. The conversion loss degrades by only about $1 \mathrm{~dB}$ at an LO power of $0 \mathrm{dBm}$.

\subsection{IF/LO/Bias Distribution}

\subsubsection{Multi-layer Routing Boards}

Each 4-pixel card contains a multi-layer printed circuit board that carries the IF, LO, and DC signals to and from four MMIC receiver modules. The routing boards are a more cost-effective and compact method for routing large numbers of signals than coaxial cables or waveguides. A summary of the design parameters for both the prototype and the production versions of the multi-layer routing boards are shown in Table 3 and the details of the multi-layer stack-ups are illustrated in Table 4. More detailed information on the prototype design are given in [15]. Figure 5 shows a CAD drawing for the production multilayer routing board. The boards have six layers: a top layer with microstrip and co-planar waveguide (red), an embedded stripline layer (purple), an embedded layer for DC lines (green), and three reference ground planes

\footnotetext{
${ }^{\ddagger}$ Thunderline-Z TL1946

${ }^{\S}$ Omnetics PSM-09-AA-M-GS-1-6 and Omnetics SSO-09-WD-18_0-C-M-GS-1-6
} 


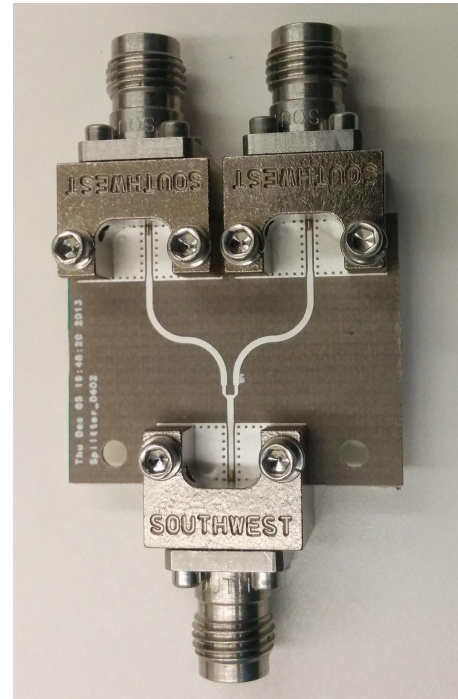

(a)

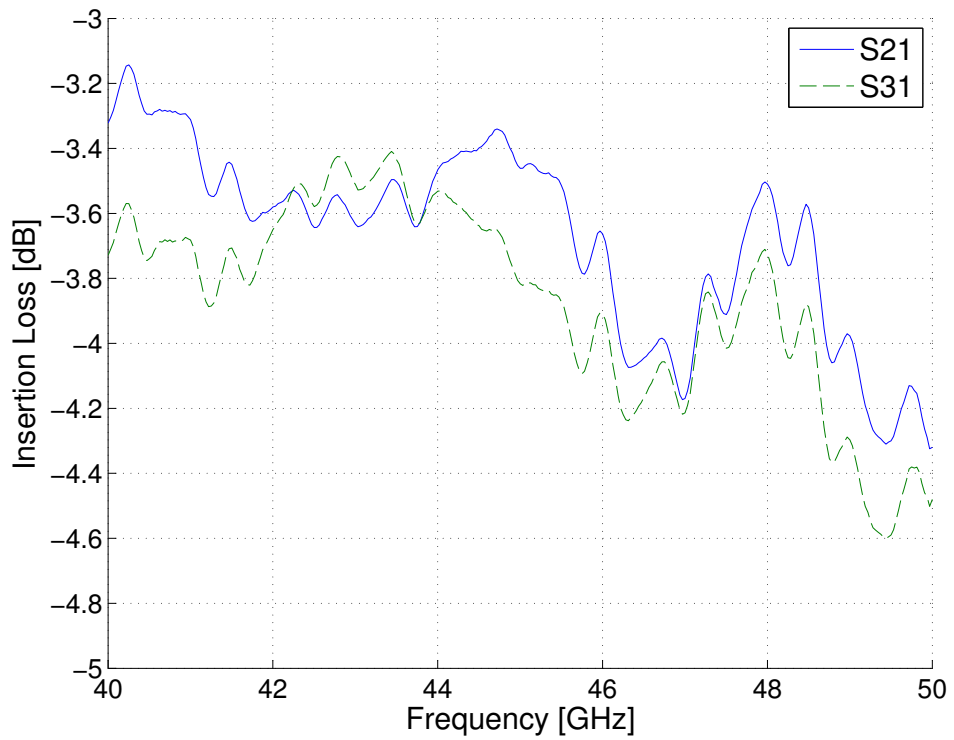

(b)

Figure 4. (a) A photograph of a Wilkinson splitter test board on 0.010" Taconic RF35-TC laminate. (b) The measured performance of the Wilkinson splitter test board. The insertion loss of the connectors and the microstrip leads were subtracted.

(not shown). The LO signal is routed on the top layer and split to the four MMIC modules, while the IF signals have a broadband transition to the interior stripline layer in order to avoid the LO lines on the top layer. The IF lines ultimately are wirebonded to the flexible circuitry, which is soldered to a ground pad on the board (§3.4.2).

One of the foremost differences between the prototype and production boards is the IF amplification scheme. Both designs utilize a commercial surface mount amplifier. The prototype implemented the broadband Hittite HMC460LC5. These devices dissipated too much power to be placed on the $20 \mathrm{~K}$ board to which the MMIC receiver modules are attached and so they were instead placed on a warmer second board that is heat sunk to the second stage of the cryostat. The production design on the other hand has a narrower IF bandwidth specification and therefore lower power devices can be utilized; this allows for the IF amplifier to be placed on the $20 \mathrm{~K}$ stage. A number of low power IF amplifier candidates are currently being evaluated.

Another difference between the two designs is the LO splitter. The prototype card utilized a rat-race coupler to split the LO. It was selected because it is straightforward to manufacture due to its planar design and lack of surface mount components. However, it is not inherently wideband; the output ports have an amplitude imbalance away from the design frequency. The production board instead uses a wideband Wilkinson splitter. A photograph of a Wilkinson splitter test board is shown in Figure 4(a) and its performance in Figure 4(b). It has adequate performance over the 40-58 GHz LO frequency range for Argus.

The challenges for implementing a multi-layer printed circuit board for Argus include achieving low loss over the LO frequencies of 40-58 GHz, providing a good impedance match at the LO connectors, and creating a balanced multi-layer stack-up that can be easily manufactured. The design approaches for addressing these challenges is outlined below.

Microstrip Loss There are three types of contributions to the microstrip insertion loss: substrate loss, metal loss, and radiation loss. The prototype card experienced unacceptably high microstrip losses at the LO frequencies; the mixer had insufficient LO power causing a severe degradation in its conversion loss (§3.3.3). Each of the three loss contributions was addressed in the production design. The substrate loss was improved in part by switching to a microwave laminate with a lower loss tangent as shown in Table 3. Additionally, the solder mask in the area around the LO traces (across a width of 0.066 ") was removed in the production designs to further improve the substrate loss. The metal loss was

${ }^{*}$ The quoted surface roughness is for the dielectric side of $0.5 \mathrm{oz} / \mathrm{ft}^{2}$ electrodeposited copper. 


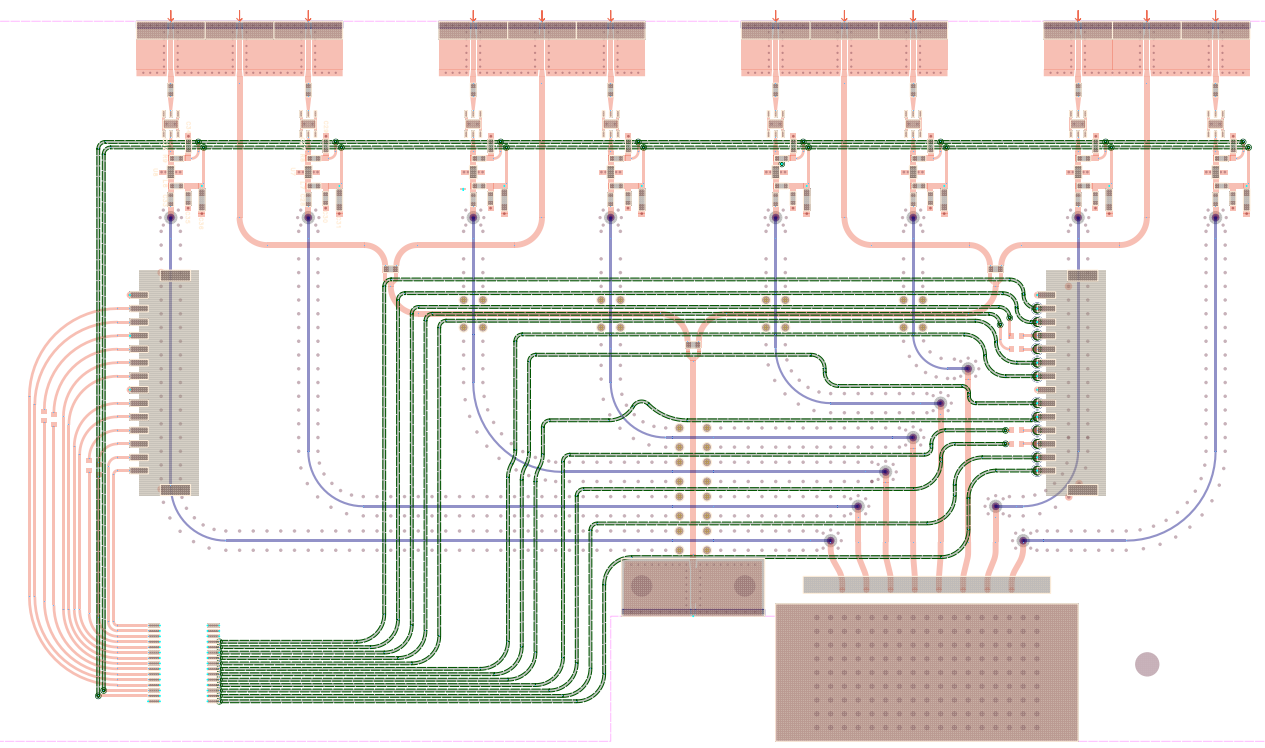

Figure 5. A CAD drawing of the production signal routing board. The board has six layers: a top layer with microstrip and co-planar waveguide (red), an embedded stripline layer (purple), an embedded layer for DC lines (green), and three reference ground planes (not shown). The pads at the top are for custom push-on connectors for the IF and LO (§3.3.1). The ground pad in the lower right is for attaching the flexible microstrip lines (§3.4.2).

\begin{tabular}{|l|c|c|}
\hline & Prototype & Production \\
\hline \hline Number of Layers & 6 & 6 \\
\hline \hline Thickness & $0.069 "$ & $0.05{ }^{\prime \prime}$ \\
\hline \hline Core & Rogers RO4350B & Taconic RF-35TC \\
\hline Material & Hydrocarbon/Ceramic & PTFE-based \\
\hline Dielectric Constant, $\epsilon_{r}$ & $3.48 @ 10 \mathrm{GHz}$ & $3.50 @ 10 \mathrm{GHz}$ \\
\hline Loss Tangent, tan $\delta$ & $0.0037 @ 10 \mathrm{GHz}$ & $0.0011 @ 10 \mathrm{GHz}$ \\
\hline Copper Surface Roughness* & $2.8 \mu \mathrm{m}$ & $0.3 \mu \mathrm{m}$ \\
\hline \hline Prepreg & Rogers RO4000 & Taconic FR-28-0040-50 \\
\hline \hline Finish & Electroless Nickel Immersion Gold (ENIG) & Immersion Silver \\
\hline
\end{tabular}

Table 3. A summary of the multilayer routing board properties for the prototype and production receivers.

\begin{tabular}{|c|c|}
\hline Prototype & Production \\
\hline \multicolumn{2}{|c|}{ Layer 1 - IF/LO Signal Lines } \\
\hline Rogers RO4350B (0.010") & Taconic RF35-TC (0.010”) \\
\hline \multicolumn{2}{|c|}{ Layer 2 - Ground Plane } \\
\hline Rogers RO4450B prepreg (0.016”) & Taconic FR-28-0040-50 prepreg (0.008") \\
\hline \multicolumn{2}{|c|}{ Layer 3 - IF Signal Lines } \\
\hline Rogers RO4350B (0.0166") & Taconic RF35-TC (0.010") \\
\hline \multicolumn{2}{|c|}{ Layer 4 - Ground Plane } \\
\hline RO4450B prepreg (0.012) & Taconic FR-28-0040-50 prepreg (0.004") \\
\hline \multicolumn{2}{|c|}{ Layer 5-DC Bias Lines } \\
\hline Rogers RO4350B (0.010) & FR4 (0.005") \\
\hline
\end{tabular}

Table 4. Details of the multilayer routing board stack-ups. The metal layers are all $0.5 \mathrm{oz} / \mathrm{ft}^{2}$ electrodeposited copper. 
addressed by moving to an immersion silver finish, which has a conductivity similar to copper. Furthermore, the microwave laminates used in the production design offered lower copper surface roughness, which also reduced metal loss. Finally, the radiation loss was improved by changing the connector launch from a microstrip launch to a coplanar waveguide launch. After making these improvements, the insertion loss for a microstrip line in the production design was measured at $0.018 \mathrm{~dB} / \mathrm{inch} / \mathrm{GHz}$, which is sufficient for achieving enough LO power in the production Argus designs.

Connector Launch The edge launch connectors on the prototype board were measured to have a poor impedance match. Specifically, the microstrip launch used in prototyping had an adequate impedance match when used in a two-layer board, but a poor match for multilayer boards. Electromagnetic simulations revealed a resonance in the gap between the connector and the board. The resonances were detuned in the production board by using a thinner board, receding the interior ground planes away from the board edge, and by using a coplanar waveguide launch instead of microstrip.

Stack-up Considerations Table 4 shows the laminates that are used to construct the multilayer routing boards. The stack-ups involve a number of two-sided boards or "cores", which have copper on both sides and are adhered together with alternating layers of prepreg. Both the prototype and production boards use a core cap construction where the outermost materials in the stack-up (i.e. the caps) are cores and the layers are fused together with a single press. In this approach the signal lines are etched directly onto the cores instead of being patterned onto foil, which leads to better impedance control. Moreover, the single press lamination is more reliable than a multi-stage pressing construction. The authors attempted to fabricate a multilayer board using a two-stage press, which was unsuccessful because the shrinkage and expansion of the copper traces during the first press led to misalignment of the traces in the second press. The delivered boards in this attempt therefore had an unexpected number of opens and shorts.

Warpage is another factor that must be considered in creating a multilayer board stack-up. Best practice dictates that a stack-up is balanced, meaning that the board is symmetrical about its mid-plane" ; a balanced stack-up minimizes the risks of warpage. However, the edge mount connectors require the boards to be sufficiently thin for high frequency operation. The stack-up for the production board in Table 4 was chosen to have a reasonable compromise between balance, which prevents warpage, and thinness, which improves the edge mount connector performance.

\subsubsection{Flexible Circuitry}

Microstrip lines on flexible circuitry provide a means for transferring IF signals between temperature stages with low thermal loading. There is one IF flexible circuit in Argus per card and each card carries eight signals. The flexible circuit substrate is 0.005 " polyimide and has a 0.0007 " thick copper cladding. The microstrip traces are 0.011 " in width with a 1.0 " reference ground plane. These dimensions yield an insertion loss of $0.076 \mathrm{~dB} / \mathrm{GHz} / \mathrm{in}$ for these lines. The cross sectional area of copper for one flexible circuit carrying eight signals is $0.0007 \mathrm{in}^{2}$, which which is comparable to semirigid cable and sufficient for providing a thermal break. More details about the flexible circuitry are given in [16].

The flexible circuitry requires custom interconnects at the multi-layer routing board and cryostat wall. At the board interface, the ground plane of the polyimide circuitry is soldered to a ground pad on the top layer of the board. The signal lines are then connected via aluminum wire bonds. A photograph of this interface is shown in Figure 7 (a) and (b). At the cryostat wall, a custom feedthrough was designed, which is shown in Figure 6 (a) and (b). The hermetic seal at the feedthroughs is provided by glass bead connectors, which are epoxied to the metal base. The signal pins are soldered to the microstrip lines on the flexible circuitry, while custom clamps provide grounding and strain relief.

\section{SYSTEM PERFORMANCE}

The prototype receiver was characterized after cooling. Figure 8(a) shows the measured gain without the MMIC receiver modules. The gain has a significant spectral slope, which is primarily due to the insertion loss of the flexible circuitry. The expected receiver gain was tabulated by summing the measured MMIC receiver module gain ${ }^{12}$ with the prototype measurement $^{* *}$ and is plotted with negative IF frequencies corresponding to the lower sideband in the frequency downconversion. These measurements demonstrate that this design concept could feasibly used to achieve tens of $\mathrm{GHz}$ of instantaneous

\footnotetext{
"In a core cap construction the mid-plane would ideally be in the center core.

${ }^{* *}$ Assuming a fixed $\mathrm{LO}$ frequency of $50 \mathrm{GHz}$
} 


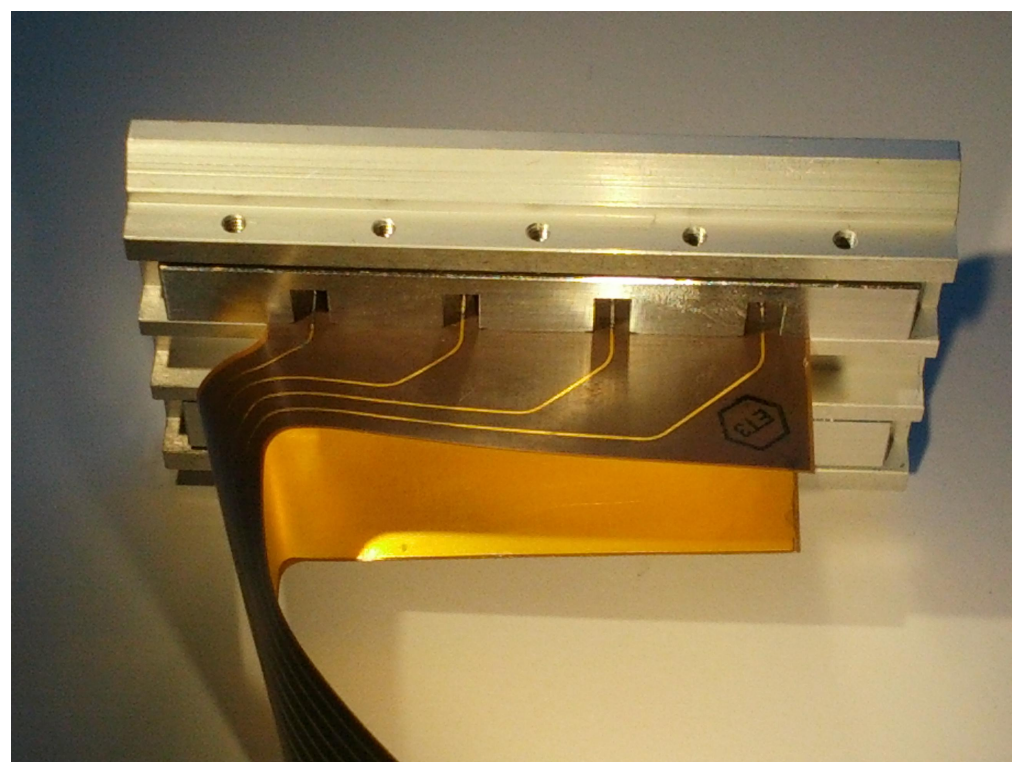

(a)

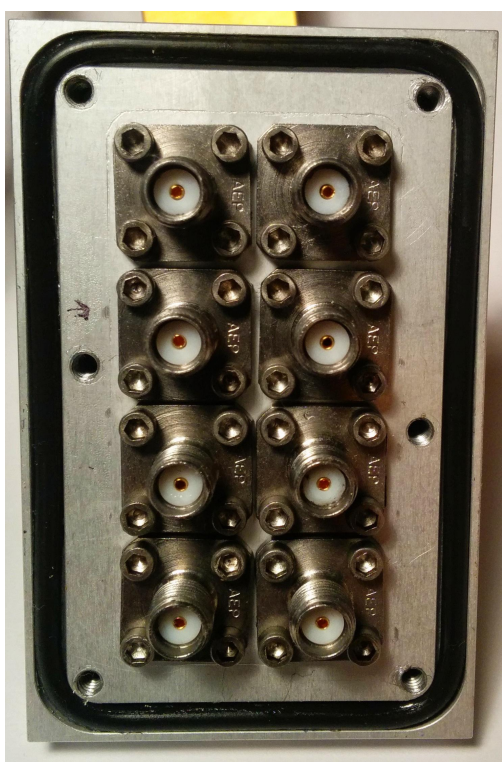

(b)

Figure 6. A hermetic feedthrough was designed for the IF signals. (a) On the inside of the cryostat the flexible microstrip lines are soldered to glass bead connectors. Clamps provide a ground connection and strain relief. (b) The IF interface on the outside of the cryostat is a pattern of 8 SMA connectors.

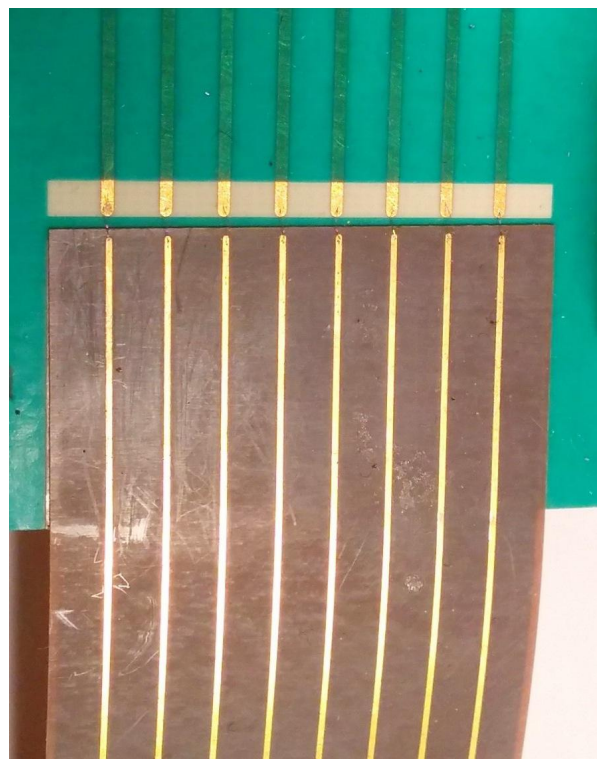

(a)

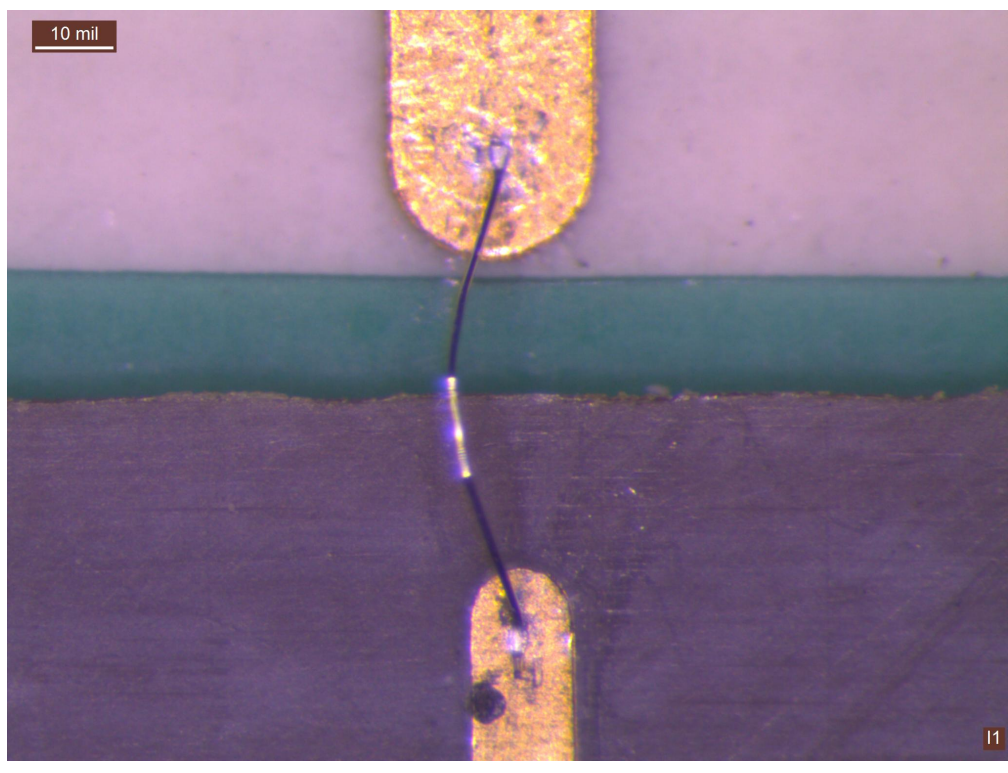

(b)

Figure 7. (a) The ground plane for the flexible microstrip lines are soldered to a ground pad on the multi-layer routing boards. Wirebonds interconnect the signal lines on the routing board and the flexible circuitry. (b) A close-up of the the wirebond interconnect. 


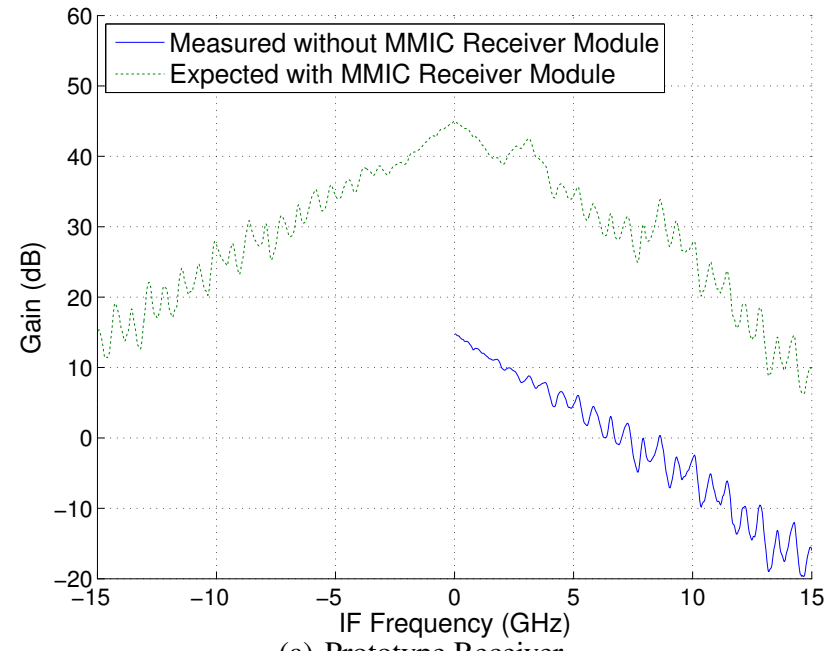

(a) Prototype Receiver

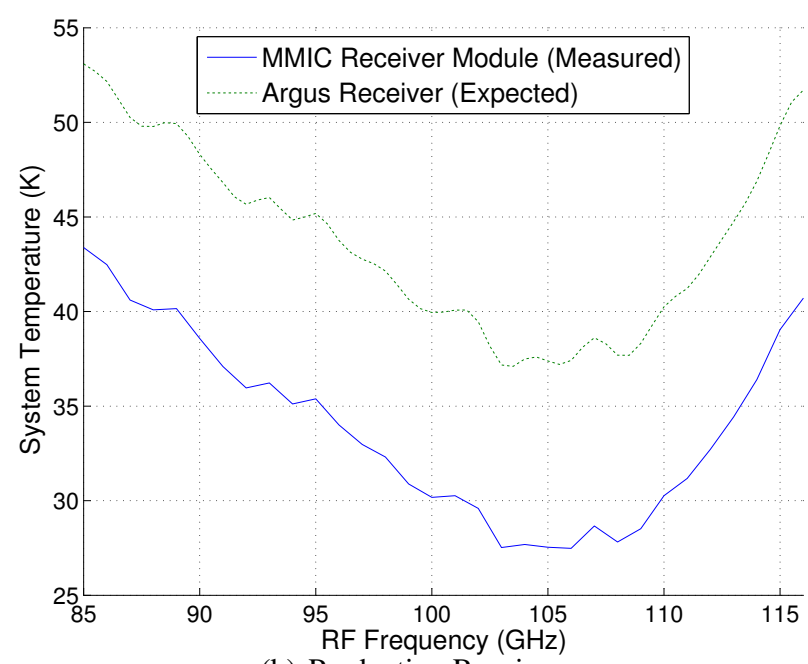

(b) Production Receiver

Figure 8. (a) The measured gain of the prototype design without the MMIC receiver module. Separate measurements of a MMIC receiver module $^{12}$ were cascaded with the prototype measurements to estimate the total cryogenic receiver gain. The negative IF frequencies correspond to the receiver's lower sideband. The prototype demonstrates the feasibility of achieving tens of GHz bandwidth with this design concept. (b) The MMIC receiver module noise temperature as reproduced from [12] as well as the expected receiver temperature for an Argus pixel.

bandwidth for future instruments. The usable bandwidth could be extended by either using another stage of IF amplification on the 4-pixel card or by using shorter transmission lines to mitigate the large spectral slope. The ripple in the measurements is from an impedance mismatch at the wirebonds ( $\$ 3.4 .2)$ and could be improved by using shorter bond lengths and/or a capacitive match to resonate out the wirebond inductance.

The expected receiver noise temperature for a production Argus pixel can be estimated by cascading measurements of individually measured sub-components. Figure 8(b) shows the MMIC receiver module noise temperature as reproduced from [12] as well as the expected receiver temperature for an Argus pixel. The window, which has not yet been measured, has been assumed to have an insertion loss of $0.1 \mathrm{~dB}$. The receiver noise temperature is below $53 \mathrm{~K}$ across the Argus band.

\section{CONCLUSIONS}

Argus, A 16-pixel millimeter-wave spectrometer, is being constructed for molecular spectroscopy in the 85-116 GHz band. Argus is scheduled for a late 2014 deployment to the Robert C. Byrd Green Bank Telescope and will become the world's fastest millimeter-wave imager. The receiver temperature for an Argus pixel is expected to be less than $53 \mathrm{~K}$.

The Argus instrument also serves as a proof of concept for receiver technology that is scalable to larger numbers of pixels. The feedhorns, MMIC receiver modules, routing boards, and flexible circuitry are all amenable to mass-production. The authors envision that this technology could be used for a future array with hundreds of pixels.

\section{ACKNOWLEDGMENTS}

The authors thank Mary Soria and Heather Owen for assembling the MMIC receiver modules.

This work was carried out in part at the Jet Propulsion Laboratory, which is operated by the California Institute of Technology for NASA. The Argus instrument is funded by NSF ATI grant 1207825 and preliminary technology development was supported by NSF ATI grant 0905855. 


\section{REFERENCES}

[1] Bryerton, E. W., Kim, Y.-M., Deal, W., Yoshida, W., Lange, M., Uyeda, J., Morgan, M., and Lai, R., “A W-band low-noise amplifier with 22K noise temperature," in [Microwave Symposium Digest, 2009. MTT '09. IEEE MTT-S International], 681-684, IEEE (2009).

[2] Samoska, L. A., Church, S., Cleary, K., Fung, A., Gaier, T. C., Kangaslahti, P., Lai, R., Lau, J. M., Mei, G., and Reeves, R., "Cryogenic MMIC Low Noise Amplifiers for W-Band and Beyond," in [International Symposium on Space Terahertz Technology], (2011).

[3] Samoska, L., Varonen, M., Reeves, R., Cleary, K., Gawande, R., Kangaslahti, P., Gaier, T., Lai, R., and Sarkozy, S., "W-Band cryogenic InP MMIC LNAs with noise below 30K," in [2012 IEEE/MTT-S International Microwave Symposium Digest ], 1-3, IEEE (June 2012).

[4] Varonen, M., Reeves, R., Kangaslahti, P., Samoska, L., Akgiray, A., Cleary, K., Gawande, R., Fung, A., Gaier, T., Weinreb, S., Readhead, A. C. S., Lawrence, C., Sarkozy, S., and Lai, R., "A 75-116-GHz LNA with 23-K noise temperature at $108 \mathrm{GHz}$," in [IEEE MTT-S International Microwave Symposium Digest (MTT)], 1-3, IEEE (2013).

[5] Erickson, N. and Narayanan, G., "MMIC Receiver Systems Developed at the University of Massachusetts, Amherst," in [MMIC Array Receivers and Spectrographs Workshop ], (2008).

[6] Narayanan, G., Heyer, M. H., Brunt, C., Goldsmith, P. F., Snell, R., and Li, D., "The Five College Radio Astronomy Observatory CO Mapping Survey of the Taurus Molecular Cloud," The Astrophysical Journal Supplement Series 177, 341-361 (July 2008).

[7] Groppi, C., Walker, C., Kulesa, C., Golish, D., Kloosterman, J., Weinreb, S., Jones, G., Bardin, J., Mani, H., Kuiper, T., Kooi, J., Lichtenberger, A., Cecil, T., Puetz, P., Narayanan, G., and Hedden, A., "Test and integration results from SuperCam: a 64-pixel array receiver for the $350 \mathrm{GHz}$ atmospheric window," in [SPIE Astronomical Telescopes + Instrumentation ], Holland, W. S. and Zmuidzinas, J., eds., 77410X-77410X-12, International Society for Optics and Photonics (July 2010).

[8] Bischoff, C., Brizius, A., Buder, I., Chinone, Y., Cleary, K., Dumoulin, R. N., Kusaka, A., Monsalve, R., Næ ss, S. K., Newburgh, L. B., Nixon, G., Reeves, R., Smith, K. M., Vanderlinde, K., Wehus, I. K., Bogdan, M., Bustos, R., Church, S. E., Davis, R., Dickinson, C., Eriksen, H. K., Gaier, T., Gundersen, J. O., Hasegawa, M., Hazumi, M., Holler, C., Huffenberger, K. M., Imbriale, W. A., Ishidoshiro, K., Jones, M. E., Kangaslahti, P., Kapner, D. J., Lawrence, C. R., Leitch, E. M., Limon, M., McMahon, J. J., Miller, A. D., Nagai, M., Nguyen, H., Pearson, T. J., Piccirillo, L., Radford, S. J. E., Readhead, A. C. S., Richards, J. L., Samtleben, D., Seiffert, M., Shepherd, M. C., Staggs, S. T., Tajima, O., Thompson, K. L., Williamson, R., Winstein, B., Wollack, E. J., and Zwart, J. T. L., “The Q/U Imaging Experiment Instrument," The Astrophysical Journal 768, 9 (May 2013).

[9] Gao, Y. and Solomon, P. M., "The Star Formation Rate and Dense Molecular Gas in Galaxies," The Astrophysical Journal 606, 271-290 (May 2004).

[10] Voll, P., Tantawi, S., and El-Beltagy, M., "A Smooth-Walled Feedhorn Antenna Design for Astrophysical Instrumentation in Space," in [Proceedings of the 62nd International Astronautical Congress], 1-7 (2011).

[11] Leong, Y.-C. and Weinreb, S., "Full band waveguide-to-microstrip probe transitions," in [Microwave Symposium Digest, 1999 IEEE MTT-S International ], 4, 1435-1438, IEEE (1999).

[12] Gawande, R., Reeves, R., Cleary, K., Kooi, J., A., R., Gaier, T., Kangaslahti, P., Samoska, L., Varonen, M., Church, S., Devaraj, K., Sieth, M., Voll, P., Harris, A., Lai, R., and Sarkozy, S., "A W-band heterodyne receiver module: a prototype for focal plane arrays," in [Microwave Symposium Digest (MTT), 2014 IEEE MTT-S International], 3-5 (2014).

[13] Russell, D., Cleary, K., and Reeves, R., "Cryogenic probe station for on-wafer characterization of electrical devices.," The Review of scientific instruments 83, 044703 (Apr. 2012).

[14] Gawande, R., Reeves, R., Samoska, L., Cleary, K., Readhead, A. C., Gaier, T., Kangaslahti, P., and Church, S., "Wband IQ sub-harmonic mixers with low LO power for cryogenic operation in large arrays," in [Microwave Conference (EuMC), 2014 44th European ], (2014).

[15] Sieth, M., Church, S., Lau, J. M., Voll, P., Gaier, T., Kangaslahti, P., Samoska, L., Soria, M., Cleary, K., Gawande, R., Readhead, A. C., Reeves, R., Harris, A., Neilson, J., Tantawi, S., and Van Winkle, D., "Technology developments for a large-format heterodyne MMIC array at W-band," International Journal of Microwave and Wireless Technologies 4, 299-307 (Apr. 2012).

[16] Harris, A. I., Sieth, M., Lau, J. M., Church, S. E., Samoska, L. A., and Cleary, K., "Note: cryogenic microstriplineon-Kapton microwave interconnects.," The Review of scientific instruments 83, 086105 (Aug. 2012). 